\title{
On Brocard Points of Harmonic Quadrangle in Isotropic Plane
}

On Brocard Points of Harmonic Quadrangle in Isotropic Plane

\section{ABSTRACT}

In this paper we present some new results on Brocard points of a harmonic quadrangle in isotropic plane. We construct new harmonic quadrangles associated to the given one and study their properties dealing with Brocard points.

Key words: isotropic plane, harmonic quadrangle, Brocard points

MSC2010: 51N25

\section{Introduction}

The geometry of harmonic quadrangle has been discussed in [3]. The harmonic quadrangle is a cyclic quadrangle $A B C D$ (see [5]) with the following property: the point of intersection of the tangents at the vertices $A$ and $C$ lies on the line $B D$, and the intersection point of the tangents at the vertices $B$ and $D$ lies on the line $A C$. If one of the requests is fulfilled, the other one automatically follows. The quadrangle $A B C D$ is chosen to have $y=x^{2}$ as a circumscribed circle and the vertices $A=\left(a, a^{2}\right), B=\left(b, b^{2}\right), C=\left(c, c^{2}\right)$, and $D=\left(d, d^{2}\right)$, with $a, b, c, d$ being mutually different real numbers, where $a<b<c<d$. In that case, sides of $A B C D$ are

$A B \ldots y=(a+b) x-a b, \quad D A \ldots y=(a+d) x-a d$,

$B C \ldots y=(b+c) x-b c, \quad A C \ldots y=(a+c) x-a c$,

$C D \ldots y=(c+d) x-c d, \quad B D \ldots y=(b+d) x-b d$.

and tangents to $y=x^{2}$ at $A, B, C, D$ are

$$
\begin{array}{ll}
\mathcal{A} \ldots y=2 a x-a^{2}, & \mathcal{B} \ldots y=2 b x-b^{2}, \\
\mathcal{C} \ldots y=2 c x-c^{2}, & \mathcal{D} \ldots y=2 d x-d^{2} .
\end{array}
$$

O Brocardovim točkama harmoničnog četverokuta u izotropnoj ravnini

\section{SAŽETAK}

U radu se prikazuju neki novi rezultati o Brocardovim točkama harmoničnog četverokuta u izotropnoj ravnini. Konstruiraju se novi harmonični četverokuti pridruženi danom četverokutu, te se proučavaju njihova svojstva vezana uz Brocardove točke.

Ključne riječi: izotropna ravnina, harmonični četverokut, Brocardove točke

Equations (1) and (2) can be found within Lemma 1 in [5]. In that paper the diagonal triangle of a cyclic quadrangle was introduced where diagonal points $U=A C \cap B D$, $V=A B \cap C D$ and $W=A D \cap B C$ are given by

$$
\begin{aligned}
& U=\left(\frac{a c-b d}{a-b+c-d}, \frac{a c(b+d)-b d(a+c)}{a-b+c-d}\right), \\
& V=\left(\frac{a b-c d}{a+b-c-d}, \frac{a b(c+d)-c d(a+b)}{a+b-c-d}\right), \\
& W=\left(\frac{a d-b c}{a-b-c+d}, \frac{a d(b+c)-b c(a+d)}{a-b-c+d}\right) .
\end{aligned}
$$

Taking $a c=b d=-k^{2}, k>0$ we deal with harmonic quadrangle in a standard position. As every harmonic quadrangle can be represented in the standard position, in order to prove geometric facts for each harmonic quadrangle, it is sufficient to give a proof for the standard harmonic quadrangle. The diagonal points given by (3) in the case of 
standard position turn into

$$
\begin{aligned}
& U=\left(0, k^{2}\right), \quad V=\left(\frac{a b-c d}{a+b-c-d},-k^{2}\right), \\
& W=\left(\frac{a d-b c}{a-b-c+d},-k^{2}\right) .
\end{aligned}
$$

Due to Theorem 1 in [3] more characterizations of harmonic quadrangles among the cyclic ones are given.

Theorem 1 Let $A B C D$ be an allowable cyclic quadrangle with vertices $A=\left(a, a^{2}\right), B=\left(b, b^{2}\right), C=\left(c, c^{2}\right)$, $D=\left(d, d^{2}\right)$, sides (1) and tangents of its circumscribed circle $y=x^{2}$ at its vertices are given by (2). These are the equivalent statements:

1. the point $T_{A C}=\mathcal{A} \cap \mathcal{C}$ is incident with the diagonal $B D$;

2. the point $T_{B D}=\mathcal{B} \cap \mathcal{D}$ is incident with the diagonal $A C$;

3. the equality

$$
d(A, B) \cdot d(C, D)=-d(B, C) \cdot d(D, A)
$$

holds;

4. the equality

$$
2(a c+b d)=(a+c)(b+d)
$$

holds.

In the sequel we will deal with Brocard points of these quadrangles defined in [3] and show several properties of them connected to the harmonic quadrangles associated to $A B C D$. For that purpose the following lemma will be very useful.

Lemma 1 Let $A B C D$ be an allowable cyclic quadrangle in the standard position with vertices $A=\left(a, a^{2}\right), B=\left(b, b^{2}\right)$, $C=\left(c, c^{2}\right), D=\left(d, d^{2}\right)$. The following equalities are valid

$$
\begin{array}{ll}
a b+k^{2}=k(b-a), & b c+k^{2}=k(c-b), \\
c d+k^{2}=k(d-c), & d a+k^{2}=k(a-d) .
\end{array}
$$

Proof. Let us prove $a b+k^{2}=k(b-a)$. Taking (see [3])

$$
\frac{(a-c)(b-d)}{a+c-b-d}=-2 k
$$

in

$-2 k(a-b)-2 a b=2 k^{2}$ we get

$\frac{-a^{2} b+a b^{2}-a^{2} d+b^{2} c-4 a b c+4 a b d+a b c-a b d+a c d-b c d}{a-b+c-d}$
$=2 k^{2}$ i.e.

$\frac{-a^{2} b+a b^{2}-a^{2} d+b^{2} c-4 k^{2}(a-b)}{a-b+c-d}+k^{2}=2 k^{2}$.

After employing $(a+c)(b+d)=-4 k^{2}$ from [3], the upper equality turns into

$\frac{-a^{2} b+a b^{2}-a^{2} d+b^{2} c+(a+c)(b+d)(a-b)}{a-b+c-d}+k^{2}=2 k^{2}$.

Immediately,

$\frac{k^{2}(a-b+c-d)}{a-b+c-d}+k^{2}=2 k^{2}$

follows and the claim of Lemma is proved.

Further on we will always deal with the harmonic quadrangle in the standard position.

\section{Brocard points}

There is an interesting result discussed in [3]: whole family of harmonic quadrangles can be obtained out of the given harmonic quadrangle. Namely, let $A B C D$ be a harmonic quadrangle. Lines $a^{\prime}, b^{\prime}, c^{\prime}, d^{\prime}$ are taken in a way to be incident to vertices $A, B, C, D$, respectively, and make equal angles to the sides $A B, B C, C D, D A$, respectively. The quadrangle formed by lines $a^{\prime}, b^{\prime}, c^{\prime}, d^{\prime}$ is a harmonic quadrangle as well. Furthermore, denoting obtained quadrangle by $A^{\prime} B^{\prime} C^{\prime} D^{\prime}$, the ratios of the corresponding sides of given quadrangle $A B C D$ and obtained quadrangle $A^{\prime} B^{\prime} C^{\prime} D^{\prime}$ are equal. Only in one case, points $A^{\prime}, B^{\prime}, C^{\prime}, D^{\prime}$ coincide with one point $P_{1}$, the first Brocard point. In similar manner, the second Brocard point $P_{2}$ is obtained as well. In the latter case lines $P_{2} A, P_{2} B, P_{2} C$, and $P_{2} D$ form the equal angles with the sides $A D, D C, C B$, and $B A$, respectively. Brocard points are of the form

$P_{1}=\left(k, 3 k^{2}\right), \quad P_{2}=\left(-k, 3 k^{2}\right)$.

The Brocard points can easily be constructed by using the fact that

$P_{1}=W M_{A C} \cap V M_{B D}, \quad P_{2}=V M_{A C} \cap W M_{B D}$

where $M_{A C}, M_{B D}$ are midpoints of the line segments $\overline{A C}$, $\overline{B D}$, respectively.

The Euclidean case of Theorem 2 can be found in [1], and the Euclidean analogue of Theorem 3 is stated in [2] and [4]. 
Theorem 2 Let $A B C D$ be a harmonic quadrangle and $P_{1}, P_{2}$ its Brocard points. The following equality holds:

$$
\begin{aligned}
& d\left(A, P_{1}\right) \cdot d\left(B, P_{1}\right) \cdot d\left(C, P_{1}\right) \cdot d\left(D, P_{1}\right) \\
& \quad=d\left(A, P_{2}\right) \cdot d\left(B, P_{2}\right) \cdot d\left(C, P_{2}\right) \cdot d\left(D, P_{2}\right) .
\end{aligned}
$$

\section{Proof. Since}

$$
\begin{aligned}
d( & \left.A, P_{1}\right) \cdot d\left(B, P_{1}\right) \cdot d\left(C, P_{1}\right) \cdot d\left(D, P_{1}\right) \\
& =(k-a)(k-b)(k-c)(k-d) \\
& =\left(k^{2}-k(a+c)+a c\right)\left(k^{2}-k(b+d)+b d\right) \\
& =k^{2}(a+c)(b+d)=-4 k^{4}
\end{aligned}
$$

and

$$
\begin{aligned}
d( & \left.A, P_{2}\right) \cdot d\left(B, P_{2}\right) \cdot d\left(C, P_{2}\right) \cdot d\left(D, P_{2}\right) \\
\quad & =(-k-a)(-k-b)(-k-c)(-k-d) \\
& =\left(k^{2}+k(a+c)+a c\right)\left(k^{2}+k(b+d)+b d\right) \\
& =k^{2}(a+c)(b+d)=-4 k^{4}
\end{aligned}
$$

the theorem is proved.

Theorem 3 Let $A B C D$ be a harmonic quadrangle and $P_{1}, P_{2}$ its Brocard points. The four points $A P_{1} \cap B P_{2}$, $B P_{1} \cap C P_{2}, C P_{1} \cap D P_{2}, D P_{1} \cap A P_{2}$ lie on a circle, that is incident with the points $U, P_{1}, P_{2}, M_{A C}$ and $M_{B D}$ as well. Furthermore, they are parallel with the midpoints of the line segments $A B, B C, C D$ and $D A$, respectively.

Proof. Due to Theorem 5 in [3] the circle incident to the points $U, P_{1}, P_{2}, M_{A C}$ and $M_{B D}$ is given by

$y=2 x^{2}+k^{2}$.

Let us take for example the point $A P_{1} \cap B P_{2}$. Lines $A P_{1}$ and $B P_{2}$ have the equations (see Theorem 4 in [3])

$y=(a+b+2 k) x-a(2 k+b)$,

$y=(b+a-2 k) x-b(-2 k+a)$.

Thus, the point $A P_{1} \cap B P_{2}$ is of the form

$$
\left(\frac{a+b}{2}, k(b-a)+\frac{a^{2}+b^{2}}{2}\right) .
$$

Due to Lemma 1 the equality $k(b-a)=a b+k^{2}$ is valid, and therefore the point $A P_{1} \cap B P_{2}$ is incident to (10).

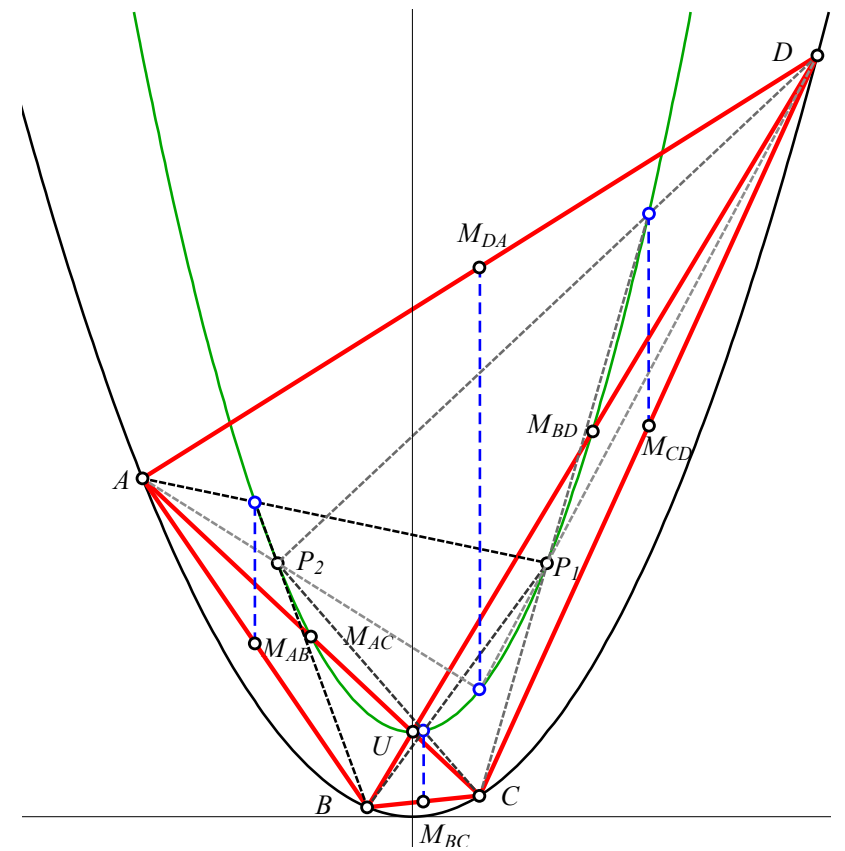

Figure 1: Visualization of Theorem 3

In the sequel we will study two quadrangles associated to the given harmonic quadrangle $A B C D$. Let $A^{i}, B^{i}, C^{i}, D^{i}$ be the intersection points of its circumscribed circle $y=x^{2}$ with the lines $A P_{i}, B P_{i}, C P_{i}, D P_{i}$, respectively, $i=1,2$. According to [3] (Theorem 4, for $h=-2 k$ ), the line $A P_{1}$ has the equation $y=(a+b+2 k) x-a(2 k+b)$. Hence, $A^{1}$ has coordinates $\left(b+2 k,(b+2 k)^{2}\right)$. Similarly, we get the other intersections:

$$
\begin{aligned}
& A^{1}=\left(b+2 k,(b+2 k)^{2}\right), \quad B^{1}=\left(c+2 k,(c+2 k)^{2}\right), \\
& C^{1}=\left(d+2 k,(d+2 k)^{2}\right), \quad D^{1}=\left(a+2 k,(a+2 k)^{2}\right),
\end{aligned}
$$

and

$$
\begin{aligned}
& A^{2}=\left(d-2 k,(d-2 k)^{2}\right), B^{2}=\left(a-2 k,(a-2 k)^{2}\right), \\
& C^{2}=\left(b-2 k,(b-2 k)^{2}\right), \quad D^{2}=\left(c-2 k,(c-2 k)^{2}\right) .
\end{aligned}
$$

Some interesting properties of the obtained quadrangles are stated in Theorems 4-9 that follow. The authors haven't found their Euclidean counterparts in the literature available to them, but they are convinced in their validity in the Euclidean plane as well.

Theorem 4 The quadrangles $A^{1} B^{1} C^{1} D^{1}$ and $A^{2} B^{2} C^{2} D^{2}$, associated to the harmonic quadrangle $A B C D$, are harmonic quadrangles as well.

Proof. From (11) we get equations (see Theorem 4 in [3]) $d\left(A^{1}, B^{1}\right) \cdot d\left(C^{1}, D^{1}\right)=(c-b)(a-d)=d(B, C) \cdot d(D, A)$, $d\left(B^{1}, C^{1}\right) \cdot d\left(D^{1}, A^{1}\right)=(d-c)(b-a)=d(C, D) \cdot d(A, B)$. 
Obviously, $d\left(A^{1}, B^{1}\right) \cdot d\left(C^{1}, D^{1}\right)=-d\left(B^{1}, C^{1}\right) \cdot d\left(D^{1}, A^{1}\right)$ precisely when $d(A, B) \cdot d(C, D)=-d(B, C) \cdot d(D, A)$. Therefore, $A^{1} B^{1} C^{1} D^{1}$ is a harmonic quadrangle. Similar procedure gives the proof for $A^{2} B^{2} C^{2} D^{2}$.

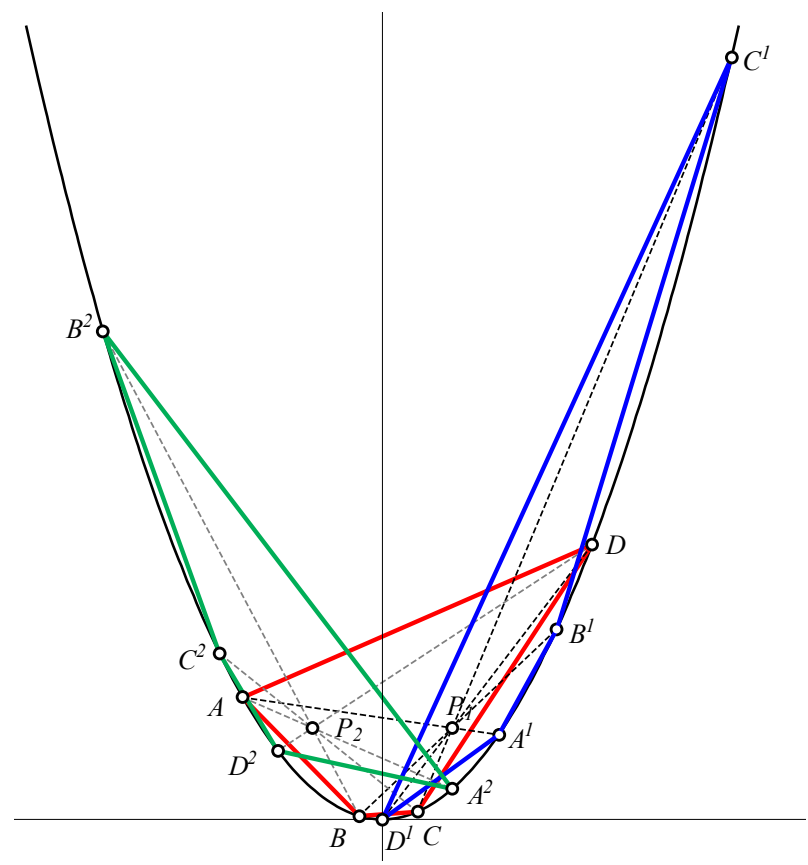

Figure 2: Harmonic quadrangles $A^{1} B^{1} C^{1} D^{1} \quad$ and $A^{2} B^{2} C^{2} D^{2}$.

Theorem 5 For the quadrangles $A^{1} B^{1} C^{1} D^{1}$ and $A^{2} B^{2} C^{2} D^{2}$ associated to the harmonic quadrangle $A B C D$ the equalities

$$
\begin{aligned}
& \angle\left(D^{2} A^{2}, A^{2} B^{2}\right)=-\angle(A B, B C)=\angle\left(B^{1} C^{1}, C^{1} D^{1}\right), \\
& \angle\left(A^{2} B^{2}, B^{2} C^{2}\right)=-\angle(B C, C D)=\angle\left(C^{1} D^{1}, D^{1} A^{1}\right), \\
& \angle\left(B^{2} C^{2}, C^{2} D^{2}\right)=-\angle(C D, D A)=\angle\left(D^{1} A^{1}, A^{1} B^{1}\right), \\
& \angle\left(C^{2} D^{2}, D^{2} A^{2}\right)=-\angle(D A, A B)=\angle\left(A^{1} B^{1}, B^{1} C^{1}\right),
\end{aligned}
$$

and

$$
\begin{aligned}
& d\left(A^{1}, B^{1}\right)=d(B, C)=d\left(C^{2}, D^{2}\right), \\
& d\left(B^{1}, C^{1}\right)=d(C, D)=d\left(D^{2}, A^{2}\right), \\
& d\left(C^{1}, D^{1}\right)=d(D, A)=d\left(A^{2}, B^{2}\right), \\
& d\left(D^{1}, A^{1}\right)=d(A, B)=d\left(B^{2}, C^{2}\right)
\end{aligned}
$$

hold.

Proof. Let us prove $\angle\left(D^{2} A^{2}, A^{2} B^{2}\right)=-\angle(A B, B C)$. From (12) we get

$$
\begin{array}{lll}
A^{2} B^{2} & \ldots & y=(d+a-4 k) x-(d-2 k)(a-2 k), \\
A^{2} D^{2} & \ldots & y=(d+c-4 k) x-(d-2 k)(c-2 k),
\end{array}
$$

and therefore, $\angle\left(D^{2} A^{2}, A^{2} B^{2}\right)=a-c$. The equality $\angle(A B, B C)=c-a$, obtained from (1), completes the first part of the proof.

The second part of the theorem follows directly from (11) and (12).

Theorem 6 For the quadrangles $A^{1} B^{1} C^{1} D^{1}$ and $A^{2} B^{2} C^{2} D^{2}$ associated to the harmonic quadrangle $A B C D$ the following statements on their diagonal points $U^{i}=$ $A^{i} C^{i} \cap B^{i} D^{i}, i=1,2$, are valid:

1. $d\left(U^{1}, U\right)=d\left(U, U^{2}\right)$.

2. The connection line $U^{1} U^{2}$ is parallel to the connection lines $P^{1} P^{2}$ and $V W$.

3. The points $U^{1}, U^{2}$ are incident with the lines $U P_{1}$, $U P_{2}$, respectively.

Proof. Directly from (11) and (12) the coordinates of $U^{1}$, $U^{2}$ are obtained to be

$U^{1}=\left(2 k, 5 k^{2}\right), \quad U^{2}=\left(-2 k, 5 k^{2}\right)$.

Thus, $d\left(U^{1}, U\right)=-2 k=d\left(U, U^{2}\right)$.

The connection line $U^{1} U^{2}$ has the equation $y=5 k^{2}$ and it is parallel to the lines $P^{1} P^{2}$ and $V W$ having the equations $y=3 k^{2}$ and $y=-k^{2}$.

The last part of the theorem holds since the coordinates of the points $U, U^{1}, P_{1}$ satisfy the equation $y=2 k x+k^{2}$, while the coordinates of the points $U, U^{2}, P_{2}$ satisfy the equation $y=-2 k x+k^{2}$.

Theorem 7 The diagonal points $V^{i}=A^{i} B^{i} \cap C^{i} D^{i}$ and $W^{i}=A^{i} D^{i} \cap B^{i} D^{i}$ of the quadrangles $A^{i} B^{i} C^{i} D^{i}$ are incident with the lines $V P_{i}$ and $W P_{i}$, respectively, $i=1,2$.

Proof. Let us prove that $V^{1}=A^{1} B^{1} \cap C^{1} D^{1}$ is incident with $V P_{1}$, i.e. $V^{1}, V, P_{1}$ are collinear points. From (3) and (11) we get

$V^{1}=\left(\frac{a d-b c}{a-b-c+d}+2 k, 4 k \frac{a d-b c}{a-b-c+d}+3 k^{2}\right)$.

Now, the slopes of the lines $V P_{1}$ and $V^{1} P_{1}$ are obtained to be

$$
\frac{-4 k^{2}(a+b-c-d)}{a b-c d-k(a+b-c-d)}, \quad \frac{4 k(a d-b c)}{a d-b c+k(a-b-c+d)},
$$

respectively, and are equal precisely when

$$
\begin{gathered}
-k(a+b-c-d)[a d-b c+k(a-b-c+d)] \\
=(a d-b c)[a b-c d-k(a+b-c-d)]
\end{gathered}
$$

i. e.

$-k^{2}(a+b-c-d)(a-b-c+d)=(a d-b c)(a b-c d)$. 
This is true since

$$
\begin{gathered}
-k^{2}\left(a^{2}-2 a c+c^{2}-b^{2}+2 b d-d^{2}\right) \\
=-a^{2} k^{2}+d^{2} k^{2}+b^{2} k^{2}-c^{2} k^{2} .
\end{gathered}
$$

The other three collinearities can be proved in a similar way.

Theorem 8 Let $M_{A^{i} C^{i}}$ and $M_{B^{i} D^{i}}$ be the midpoints of the line segments $A^{i} C^{i}$ and $B^{i} D^{i}$, respectively, $i=1,2$. Then the following quadruples of points are collinear: $\left\{M_{A^{1} C^{1}}, V, M_{B D}, P_{1}\right\},\left\{M_{B^{1} D^{1}}, W, M_{A C}, P_{1}\right\}$, $\left\{M_{A^{2} C^{2}}, W, M_{B D}, P_{2}\right\},\left\{M_{B^{2} D^{2}}, V, M_{A C}, P_{2}\right\}$.

Proof. Let us prove the claim of Theorem for the quadruple of points $\left\{M_{A^{1} C^{1}}, V, M_{B D}, P_{1}\right\}$. According to (9) the points $V, M_{B D}, P_{1}$ are collinear. Therefore, it is sufficient to show that the points $M_{B D}\left(\frac{b+d}{2}, \frac{b^{2}+d^{2}}{2}\right), M_{A^{1} C^{1}}\left(\frac{b+d+4 k}{2}, \frac{(b+2 k)^{2}+(d+2 k)^{2}}{2}\right)$, and $P_{1}\left(k, 3 k^{2}\right)$ are collinear. Their coordinates satisfy the equation $y=(b+d+2 k) x-(b+d) k+k^{2}$.

Indeed,

$$
\begin{aligned}
(b & +d+2 k) \frac{b+d}{2}-(b+d) k+k^{2} \\
& =\frac{(b+d)^{2}}{2}+(b+d) k-(b+d) k+k^{2}=\frac{b^{2}+d^{2}}{2} .
\end{aligned}
$$

Similarly,

$$
\begin{aligned}
(b & +d+2 k) \frac{b+d+4 k}{2}-(b+d) k+k^{2} \\
& =\frac{(b+d)^{2}}{2}+2 k(b+d)+k(b+d)+4 k^{2}-(b+d) k+k^{2} \\
& =\frac{(b+2 k)^{2}+(d+2 k)^{2}}{2}
\end{aligned}
$$

and

$(b+d+2 k) k-(b+d) k+k^{2}=3 k^{2}$.

The other three collinearities can be proved in a similar way.

Theorem 9 Let $P_{1}^{i}$ and $P_{2}^{i}$ be Brocard points of the quadrangle $A^{i} B^{i} C^{i} D^{i}, i=1,2$. Then the second Brocard point $P_{2}^{1}$ of the quadrangle $A^{1} B^{1} C^{1} D^{1}$ coincides with $P_{1}$, while the first Brocard point $P_{1}^{2}$ of the quadrangle $A^{2} B^{2} C^{2} D^{2}$ coincides with $P_{2}$.

Proof. According to (9), $P_{2}^{1}=V^{1} M_{A^{1} C^{1}} \cap W^{1} M_{B^{1} D^{1}}$. Now, from Theorems 7-8 we get $P_{2}^{1}=V M_{B D} \cap W M_{A C}=P_{1}$. Similarly,

$P_{1}^{2}=W^{2} M_{A^{2} C^{2}} \cap V^{2} M_{B^{2} D^{2}}=W M_{B D} \cap V M_{A C}=P_{2}$.
By using two Brocard points of the given quadrangle $A B C D$ we constructed two new quadrangles. We can continue that procedure and, by using the Brocard points of the obtained quadrangles, construct further four quadrangles. Actually, we will get only two new quadrangles, since two of them coincide with the referent quadrangle $A B C D$. Indeed, if we use $A^{1} B^{1} C^{1} D^{1}$ and its first Brocard point, we will get a new quadrangle, but if we use its second Brocard point, which is $P_{1}$, then we will get $A B C D$. So, in each step of this procedure we have to use the first Brocard points, or we always have to use the second Brocard points.

Some computations verify that if we start a pattern with the quadrangle $A B C D$ in the step 0 , and the quadrangle $A^{1} B^{1} C^{1} D^{1}$ in the step 1 , then the first Brocard point in the step $n$ has the coordinates $\left((2 n+1) k,(2 n+1)^{2} k^{2}+2 k^{2}\right)$. Therefore, we conclude that all Brocard points of the quadrangles associated to the quadrangle $A B C D$ lie on the circle $y=x^{2}+2 k^{2}$.

We will now focus on the other two quadrangles associated to the given harmonic quadrangle $A B C D$. Let the quadrangles $A^{3} B^{3} C^{3} D^{3}$ and $A^{4} B^{4} C^{4} D^{4}$ be defined in the following way: The lines $l_{A B}, l_{B C}, l_{C D}, l_{D A}$ incident with the diagonal point $U$ and parallel to $A B, B C, C D, D A$, respectively, intersect the sides of the quadrangle $A B C D$ in eight points

$$
\begin{array}{ll}
A^{3}=l_{A B} \cap A D, \\
B^{3}=l_{B C} \cap A B, \\
C^{3}=l_{C D} \cap B C, & A^{4}=l_{A D} \cap A B, \\
D^{3}=l_{A D} \cap C D, & B^{4}=l_{A B} \cap B C, \\
& D^{4}=l_{C D} \cap C D,
\end{array}
$$

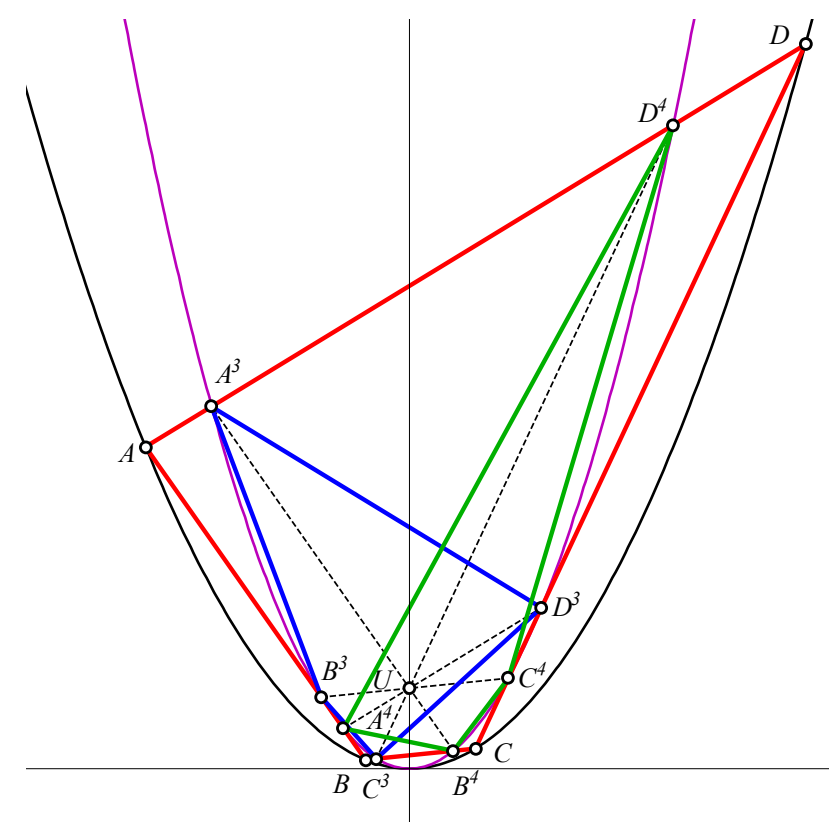

Figure 3: Harmonic quadrangles $A^{3} B^{3} C^{3} D^{3}$ and $A^{4} B^{4} C^{4} D^{4}$ 
It was shown in [3] that the constructed eight points lie on a circle $y=2 x^{2}$. The properties of the quadrangles $A^{3} B^{3} C^{3} D^{3}$ and $A^{4} B^{4} C^{4} D^{4}$ in the Euclidean plane have been discussed in [4]. Here we present the similar study for the isotropic plane.

Since the lines $l_{A B}$ and $A D$ are given by

$l_{A B} \quad \ldots \quad y=(a+b) x+k^{2}$,

$A D \quad \ldots \quad y=(a+d) x-a d$,

the first coordinate of their intersection point $A^{3}$ is $\frac{a d+k^{2}}{d-b}$. According to Lemma 1, it equals $\frac{k(a-d)}{d-b}$. Similarly, all intersection points are obtained as

$$
\begin{aligned}
A^{3} & =\left(\frac{k(a-d)}{d-b}, 2\left(\frac{k(a-d)}{d-b}\right)^{2}\right), \\
B^{3} & =\left(\frac{k(b-a)}{a-c}, 2\left(\frac{k(b-a)}{a-c}\right)^{2}\right), \\
C^{3} & =\left(\frac{k(c-b)}{b-d}, 2\left(\frac{k(c-b)}{b-d}\right)^{2}\right), \\
D^{3} & =\left(\frac{k(d-c)}{c-a}, 2\left(\frac{k(d-c)}{c-a}\right)^{2}\right)
\end{aligned}
$$

and

$$
\begin{aligned}
& A^{4}=\left(\frac{k(b-a)}{b-d}, 2\left(\frac{k(b-a)}{b-d}\right)^{2}\right), \\
& B^{4}=\left(\frac{k(c-b)}{c-a}, 2\left(\frac{k(c-b)}{c-a}\right)^{2}\right), \\
& C^{4}=\left(\frac{k(d-c)}{d-b}, 2\left(\frac{k(d-c)}{d-b}\right)^{2}\right), \\
& D^{4}=\left(\frac{k(a-d)}{a-c}, 2\left(\frac{k(a-d)}{a-c}\right)^{2}\right) .
\end{aligned}
$$

Theorem 10 For the quadrangles $A^{i} B^{i} C^{i} D^{i}, i=3,4$, the equalities

$$
\frac{d\left(A^{i}, B^{i}\right)}{d(A, B)}=\frac{d\left(B^{i}, C^{i}\right)}{d(B, C)}=\frac{d\left(C^{i}, D^{i}\right)}{d(C, D)}=\frac{d\left(D^{i}, A^{i}\right)}{d(D, A)}=\frac{1}{2}
$$

and

$$
\begin{aligned}
& \angle\left(D^{i} A^{i}, A^{i} B^{i}\right)=\angle(D A, A B), \\
& \angle\left(A^{i} B^{i}, B^{i} C^{i}\right)=\angle(A B, B C), \\
& \angle\left(B^{i} C^{i}, C^{i} D^{i}\right)=\angle(B C, C D), \\
& \angle\left(C^{i} D^{i}, D^{i} A^{i}\right)=\angle(C D, D A)
\end{aligned}
$$

hold.
Proof. In order to show (14) is valid, we give a proof for $\frac{d\left(A^{3}, B^{3}\right)}{d(A, B)}=\frac{1}{2}$. Indeed,

$$
\begin{aligned}
& d\left(A^{3}, B^{3}\right)=\frac{k(b-a)}{a-c}+\frac{k(a-d)}{b-d} \\
& \quad=k \frac{(b-a)(b-d)+(a-c)(a-d)}{(a-c)(b-d)} \\
& \quad=k \frac{(a-b)(a-b+c-d)}{(a-c)(b-d)}=k \frac{(a-b)(a-b+c-d)}{-2 k(a-b+c-d)} \\
& \quad=\frac{b-a}{2}=\frac{d(A, B)}{2}
\end{aligned}
$$

Further on, let us prove $\angle\left(A^{3} B^{3}, B^{3} C^{3}\right)=\angle(A B, B C)$. Out of (13) the slopes of the lines $A^{3} B^{3}$ and $B^{3} C^{3}$ are obtained to be

$2 k\left(\frac{a-d}{d-b}+\frac{b-a}{a-c}\right), \quad 2 k\left(\frac{b-a}{a-c}+\frac{c-b}{b-d}\right)$,

respectively. Thus,

$$
\begin{aligned}
& \angle\left(A^{3} B^{3}, B^{3} C^{3}\right)=\frac{2 k(a-b+c-d)}{b-d}=\frac{-(a-c)(b-d)}{b-d} \\
& \quad=c-a=\angle(A B, B C) .
\end{aligned}
$$

Theorem 11 The quadrangles $A^{3} B^{3} C^{3} D^{3}$ and $A^{4} B^{4} C^{4} D^{4}$ are harmonic quadrangles as well.

Proof. It follows directly from (14) and Theorem 1.

Theorem 12 Let $U^{i}=A^{i} C^{i} \cap B^{i} D^{i}$, be the diagonal points of the quadrangles $A^{i} B^{i} C^{i} D^{i}, i=3,4$. The following statements are valid:

1. The diagonal point $U$ is the midpoint of the line segment $U^{3} U^{4}$.

2. The connection line $U^{3} U^{4}$ is parallel to the connection lines $P_{1} P_{2}$ and $V W$.

Proof. From (14) we get the equation of the line $A^{3} C^{3}$

$y=-\frac{2 k(a+b-c-d)}{b-d} x+\frac{-2 k^{2}(b-c)(a-d)}{(b-d)^{2}}$

By using $2(a-d)(b-c)=(a-c)(b-d)$, it turns into

$y=-\frac{2 k(a+b-c-d)}{b-d} x-\frac{k^{2}(a-c)}{b-d}$.

Similarly, we get the equation of the line $B^{3} D^{3}$ as

$y=-\frac{2 k(a-b-c+d)}{a-c} x-\frac{k^{2}(b-d)}{a-c}$. 
Therefore, their intersection has coordinates $U^{3}=$ $\left(-\frac{k}{2}, k^{2}\right)$. Analogously, the diagonal point $U^{4}=A^{4} C^{4} \cap$ $B^{4} D^{4}=\left(\frac{k}{2}, k^{2}\right)$ is obtained. The point $U\left(0, k^{2}\right)$ is obviously the midpoint of the points $U^{3}$ and $U^{4}$.

The line $U^{3} U^{4}$ is given by the equation $y=k^{2}$, and therefore, parallel to the connection lines $P_{1} P_{2}$ and $V W$.

Theorem 13 Let $V^{i}=A^{i} B^{i} \cap C^{i} D^{i}, W^{i}=A^{i} D^{i} \cap B^{i} D^{i}$ be the diagonal points of the quadrangles $A^{i} B^{i} C^{i} D^{i}, i=3,4$. Then the points $V^{3}, W^{4}$ are incident with $B D$, and the points $V^{4}, W^{3}$ are incident with $A C$.

Proof. To prove the theorem, we will show that $V^{3}$ lies on $B D$. The other statements can be shown in the similar manner.

Out of (13), elementary, but long calculation results with coordinates of $V^{3}=A^{3} B^{3} \cap C^{3} D^{3}$ in the form

$V^{3}=\left(\frac{c-a}{a+b-c-d} k, \frac{a-b-c+d}{a+b-c-d} k^{2}\right)$

The equality $a b-b c-c d+d a=2 k(b-d)$ that can be obtained from Lemma 1 will be used to prove that coordinates given by (15) satisfy the equation $y=(b+d) x-b d$ of the line $B D$. Indeed,

$$
\begin{aligned}
(b+d) \frac{(c-a) k}{a+b-c-d}-b d=\frac{(b c-a b+c d-a d) k}{a+b-c-d}+k^{2} \\
\quad=\frac{-2 k^{2}(b-d)}{a+b-c-d}+k^{2}=\frac{a-b-c+d}{a+b-c-d} k^{2} .
\end{aligned}
$$

Theorem 14 Let $M_{A^{i} C^{i}}$ and $M_{B^{i} D^{i}}$ be the midpoints of the line segments $A^{i} C^{i}$ and $B^{i} D^{i}$, respectively, $i=3,4$. Then $M_{A^{i} C^{i}}$ are incident with $A C$, and $B^{i} D^{i}$ are incident with $B D$, $i=3,4$.

Proof. Let us for example prove that $M_{A^{3} C^{3}}$ is incident with $A C$. It is sufficient to prove that the coordinates of the midpoint

$$
M_{A^{3} C^{3}}=\left(\frac{a+b-c-d}{d-b} \frac{k}{2}, \frac{(a-d)^{2}+(c-b)^{2}}{(d-b)^{2}} k^{2}\right)
$$

satisfy the equation $y=(a+c) x-a c$ of the line $A C$. Indeed,

$$
\frac{(a+c)(a+b-c-d) k}{2(d-b)}+k^{2}=k^{2} \frac{(a-d)^{2}+(c-b)^{2}}{(d-b)^{2}}
$$

precisely when

$$
\frac{(a+c)(a+b-c-d)}{2(d-b)} k=k^{2} \frac{(a-d)^{2}+(c-b)^{2}-(d-b)^{2}}{(d-b)^{2}} \text {. }
$$

This is true if and only if

$$
\frac{(a+c)(a+b-c-d)}{d-b}=2 k \frac{(a-d)^{2}+(c-b)^{2}-(d-b)^{2}}{(d-b)^{2}},
$$

which is, by using (7), equivalent to

$$
\begin{aligned}
& (a+c)(a+b-c-d)(a-b+c-d) \\
& \quad=(a-c)\left(a^{2}+c^{2}-2 a d-2 b c+2 b d\right) .
\end{aligned}
$$

Taking $a c=b d=-k^{2}$, we get

$(a+c)\left(d^{2}-b^{2}\right)=-4 k^{2}(d-b)$.

This is valid due to $(a+c)(b+d)=-4 k^{2}$.

Theorem 15 Let $P_{1}^{i}$ and $P_{2}^{i}$ be Brocard points of the quadrangle $A^{i} B^{i} C^{i} D^{i}, i=3,4$. Then $P_{1}^{3}=U, P_{2}^{3}=P_{2}$ and $P_{1}^{4}=P_{1}, P_{2}^{4}=U$.

Proof. The facts $P_{1}^{3}=U$ and $P_{2}^{4}=U$ follow directly from Theorems 13 and 14. Indeed, $P_{1}^{3}=W^{3} M_{A^{3} C^{3}} \cap V^{3} M_{B^{3} D^{3}}=$ $A C \cap B D=U$ and $P_{2}^{4}=V^{3} M_{A^{3} C^{3}} \cap W^{3} M_{B^{3} D^{3}}=B D \cap A C=$ $U$.

It is left to prove $P_{2}^{3}=P_{2}$ and $P_{1}^{4}=P_{1}$. For the illustration, we will prove $P_{2}^{3}=P_{2}$. It is sufficient to show that $P_{2}$ lies both on $V^{3} M_{A^{3} C^{3}}$ and $W^{3} M_{B^{3} D^{3}}$. Let us check that it lies on $V^{3} M_{A^{3} C^{3}}$, i. e. that $P_{2}, V^{3}, M_{A^{3} C^{3}}$ are collinear points.

From (8) and (15) we get the following equation of the line $P_{2} V^{3}$ :

$y=\frac{-2 k(a+2 b-c-2 d)}{b-d}(x+k)+3 k^{2}$.

From

$$
\begin{aligned}
& \frac{-2 k(a+2 b-c-2 d)}{b-d}\left(\frac{a+b-c-d}{d-b} \frac{k}{2}+k\right)+3 k^{2} \\
& =\frac{k^{2}}{(b-d)^{2}}(a+2 b-c-2 d)(a-b-c+d)+3 k^{2} \\
& =\frac{k^{2}}{(b-d)^{2}}\left(a^{2}+b^{2}+c^{2}+d^{2}-2 a c-2 b d+a b-a d-b c+c d\right) \\
& =\frac{k^{2}}{(b-d)^{2}}\left(a^{2}-2 a d+d^{2}+b^{2}-2 b c+c^{2}\right) \\
& =\frac{k^{2}}{(b-d)^{2}}\left((a-d)^{2}+(b-c)^{2}\right)
\end{aligned}
$$

we conclude that the midpoint $M_{A^{3} C^{3}}$ with coordinates (16) is incident with the line $P_{2} V^{3}$.

We can start a construction of a sequence of quadrangles with the quadrangle $A B C D$ in step 0 , and quadrangle $A^{4} B^{4} C^{4} D^{4}$ in step 1 . Some computations verify that a quadrangle constructed in step $n$ has Brocard points $\left(k, 3 k^{2}\right)$ and $\left(\left(1-\frac{1}{2^{n-1}}\right) k,\left(3-\frac{n}{2^{n-2}}\right) k^{2}\right)$. Obviously, all quadrangles in the sequence have the same first Brocard point $P_{1}=\left(k, 3 k^{2}\right)$, while their second Brocard points approach the point $P_{1}$. 


\section{References}

[1] A. Bernhart, Polygons of Pursuit, Scripta Math. 24 (1959), 23-50.

[2] F.G.W. BRown, The Brocard and Tucker Circles of a Cyclic Quadrangles, Proc. Edinb. Math. Soc. 36 (1917), 61-83.

[3] E. Jurkin, M. ŠImić Horvath, V. Volenec, J. BEBAN-BRKIĆ, Harmonic Quadrangle in Isotropic Plane, Turk. J. Math., to appear.

[4] E.M. Langley, A Note on Tucker's Harmonic Quadrilateral, Math. Gaz. 11 (1923), 306-309.

[5] V. Volenec, J. Beban-Brkić, M. Šimić, Cyclic Quadrangle in the Isotropic Plane, Sarajevo J. Math. 7(20) (2011), 265-275.

\section{Ema Jurkin}

orcid.org/0000-0002-8658-5446

e-mail: ema.jurkin@rgn.hr

Faculty of Mining, Geology and Petroleum Engineering, University of Zagreb

Pierottijeva 6, HR-10000 Zagreb, Croatia

Marija Šimić Horvath

orcid.org/0000-0001-9190-5371

e-mail: marija.simic@arhitekt.hr

Faculty of Architecture, University of Zagreb

Kačićeva 26, HR-10000 Zagreb, Croatia

Vladimir Volenec

e-mail: volenec@math.hr

Faculty of Science, University of Zagreb

Bijenička cesta 30, HR-10000 Zagreb, Croatia 